\title{
LINEAR INDEPENDENCE OF TIME FREQUENCY TRANSLATES FOR SPECIAL CONFIGURATIONS
}

\author{
Ciprian Demeter
}

Abstract. We prove that for any 4 points in the plane that belong to 2 parallel lines, there is no linear dependence between the associated time-frequency translates of any nontrivial Schwartz function. If mild Diophantine properties are satisfied, we also prove linear independence in the category of $L^{2}(\mathbb{R})$ functions.

\section{Introduction}

The following conjecture, known as the HRT conjecture appears in [4]. See also [3] for an ample discussion on the subject.

Conjecture 1.1. Let $\left(t_{j}, \xi_{j}\right)_{j=1}^{n}$ be $n \geq 2$ distinct points in the plane. Then there is no nontrivial $L^{2}$ function $f: \mathbb{R} \rightarrow \mathbb{C}$ satisfying a nontrivial linear dependence

$$
\sum_{j=1}^{n} d_{i} f\left(x+t_{j}\right) e^{2 \pi i \xi_{j} x}=0,
$$

for a.e. $x \in \mathbb{R}$.

The Conjecture was proved when $\left(t_{i}, \xi_{i}\right)_{i=1}^{n}$ sit on a lattice [5]. See also [1], [2], for alternative arguments. In particular, this is the case with any 3 points. The conjecture also follows trivially when all points are collinear. No other cases seem to appear in the literature. The following weaker conjecture has also been circulated (see for example [3]).

Conjecture 1.2. Let $\left(t_{j}, \xi_{j}\right)_{j=1}^{n}$ be $n \geq 2$ distinct points in the plane. Then there is no nontrivial Schwartz function $f: \mathbb{R} \rightarrow \mathbb{C}$ satisfying a nontrivial linear dependence

$$
\sum_{j=1}^{n} d_{i} f\left(x+t_{j}\right) e^{2 \pi i \xi_{j} x}=0,
$$

for a.e. $x \in \mathbb{R}$.

In light of the discussion above, this conjecture also follows for the lattice and when the points are collinear. No other result seems to have appeared in the direction of this conjecture.

We will call an $(n, m)$ configuration, any collection of $n+m$ distinct points in the plane, such that there exist 2 distinct parallel lines such that one of them contains

Received by the editors March 21, 2010.

The author is supported by a Sloan Research Fellowship and by NSF Grants DMS-0742740 and 0901208.

AMS subject classification: Primary 26A99; Secondary 11K70, 65Q20. 
exactly $n$ of the points, and the other one contains exactly $m$ of the points. Our main results are:

Theorem 1.3. Conjecture 1.2 holds for all $(1,3)$ and $(2,2)$ configurations.

Let $\|x\|$ denote the distance of $x$ to the nearest integer.

Theorem 1.4. Conjecture 1.1 holds for special $(1,3)$ configurations

$$
(0,0),(1,0),(1, \alpha),(1, \beta)
$$

(a) if there exists $\gamma>1$ such that

$$
\liminf _{n \rightarrow \infty} n^{\gamma} \min \left\{\left\|n \frac{\beta}{\alpha}\right\|,\left\|n \frac{\alpha}{\beta}\right\|\right\}<\infty
$$

(b) if at least one of $\alpha, \beta$ is rational

In either case, no nontrivial solution $f$ can exist satisfying minimal decay

$$
\lim _{\substack{|n| \rightarrow \infty \\ n \in \mathbb{Z}}}|f(x+n)|=0 \text {, a.e. } x
$$

Theorem 1.5. Conjecture 1.1 holds for special $(2,2)$ configurations

$$
(0,0),(1,0),(0, \alpha),(1, \beta)
$$

(a) if

$$
\liminf _{n \rightarrow \infty} n \log n \min \left\{\left\|n \frac{\beta}{\alpha}\right\|,\left\|n \frac{\alpha}{\beta}\right\|\right\}<\infty
$$

(b) if at least one of $\alpha, \beta$ is rational

In either case, no nontrivial solution $f$ can exist satisfying minimal decay

$$
\lim _{\substack{|n| \rightarrow \infty \\ n \in \mathbb{Z}}}|f(x+n)|=0 \text {, a.e. } x
$$

Unlike the approaches in [4], [5], [1], [2], the approach here is mostly number theoretical. An old theorem of Khinchine guarantees that (Lebesgue) almost every $x$ satisfies

$$
\liminf _{n \rightarrow \infty} n \log n\|n x\|<\infty,
$$

and thus (via metaplectic transforms) Conjecture 1.1 holds for "almost every" $(2,2)$ configuration. Note also that Theorem 1.4(b) answers Conjecture 9.2 (b) from [3].

Theorem 1.3 is proved by first reducing to special configurations. This is done via applying the area preserving affine transformations -also called metaplectic transformsof the plane (such as translations, rotations, shears, and area one rescalings). See Section 2 in [4] for a discussion on this.

The key feature of any special $(n, m)$ configuration of points, is the fact that any linear dependence between the corresponding time frequency translates gives rise to a recurrence along $\mathbb{Z}$ orbits $x+\mathbb{Z}$. We use Diophantine approximation to identify appropriate scales. For each fixed scale, we investigate the recurrence along finite portions of two carefully chosen distinct orbits, with length comparable to the scale.

The (2-2) case is quite simple. The two rotations by $\alpha$ and $\beta$ do not interact strongly, and hence they can be dealt with by different methods. The two orbits are selected in such a way that the trigonometric polynomials associated with $\alpha$ take conjugate values along the two orbits. This means that in absolute value, the 
contributions coming from these polynomials are identical, for the two orbits. We will refer to this as the conjugates trick. The contributions coming from the polynomials associated with $\beta$ are then compared via diophantine approximation and Riemann sums, if $\beta$ is irrational, and using a periodicity argument, if $\beta$ is rational.

The $(1,3)$ case is significantly harder, partly because the behavior of the relevant polynomial $p(x, y)=C_{0}+C_{1} e(x)+C_{2} e(y)$ near its zeros is more complicated. Our argument relies in part on the almost periodicity for trigonometric polynomials. This in turn has behind the existence of simultaneous approximants $P \in \mathbb{R}$ such that

$$
P \max \{\|P \alpha\|,\|P \beta\|\} \lesssim 1
$$

The almost periods we get become better as $\frac{\alpha}{\beta}$ gets less Diophantine (that is better approximable by rationals). The main idea of this approach is to compare the products of polynomials along the two orbits via estimates for their arithmetic means (see for example Proposition 2.4). While the products along a fixed orbit can fluctuate a lot, and thus are very difficult to control, proving upper bounds for their averages turns out to be a less complicated proposal. This requires a deeper understanding of the geometry of the points $(\{n \alpha\},\{n \beta\})$, as observed in the proof of Lemma 2.3 and Lemma 4.2. The key is that the more Diophantine $\frac{\alpha}{\beta}$ is, the more "regular" is the counting measure of the points $(\{n \alpha\},\{n \beta\})$, and this will serve as a compensation.

\section{Proof of Theorem 1.3 for $(1,3)$ configurations}

Define $[x],\{x\},\|x\|$ to be the integer part, the fractional part and the distance to the nearest integer of $x$. Let $\langle x\rangle$ denote the unique number in $[-1 / 2,1 / 2)$ such that $x-\langle x\rangle$ is an integer. For two quantities $A, B$ that vary, we will denote by $A \lesssim B$ or $A=O(B)$ the fact that $A \leq C B$ for some universal constant $C$, independent of $A$ and $B$. In general, $A \lesssim_{p} B$ means that the implicit constant is allowed to depend on the parameter $p$. If no parameter is specified, the implicit constants are implicitly understood to depend on the (harmless) fundamental parameters introduced in the beginning of the proof of Theorem 1.3. For a set $A \subset \mathbb{R}$, we will denote by $|A|$ its Lebesgue measure, and if the set is finite, $|A|$ will represent its cardinality. Finally, we define $e(x):=e^{2 \pi i x}$.

We prove a few results that will contribute to the proof of Theorem 1.3.

Lemma 2.1. Let $C_{0}, C_{1}, C_{2} \in \mathbb{C}$ be some nonzero complex numbers. The polynomial $p(x, y)=C_{0}+C_{1} e(x)+C_{2} e(y)$ has at most two real zeros $\left(\gamma_{1}^{(j)}, \gamma_{2}^{(j)}\right) \in[0,1)^{2}, j \in$ $\{1,2\}$. There exists $t=t\left(C_{0}, C_{1}, C_{2}\right) \in \mathbb{R} \backslash 0$ such that

$$
|p(x, y)| \gtrsim_{C_{0}, C_{1}, C_{2}} \min _{j}\left(\left\|x-\gamma_{1}^{j}+t\left\langle y-\gamma_{2}^{j}\right\rangle\right\|+\left\|x-\gamma_{1}^{j}\right\|^{2}+\left\|y-\gamma_{2}^{j}\right\|^{2}\right),
$$

for each $x, y \in \mathbb{R}$.

Proof. If $\left|C_{0}\right|,\left|C_{1}\right|,\left|C_{2}\right|$ cannot form a triangle, then $|p(x, y)| \gtrsim_{C_{0}, C_{1}, C_{2}} 1$ and there is nothing to prove. If $\left|C_{0}\right|,\left|C_{1}\right|,\left|C_{2}\right|$ can form a triangle, given that the side with length $\left|C_{0}\right|$ is rigid, there are only two possible ways to construct the other two sides (the two triangles will be symmetric with respect to the side with length $\left|C_{0}\right|$ ). This justifies the fact that there are at most 2 zeros. 
Since $p,\|\cdot\|$ and $\langle\cdot\rangle$ are 1 periodic, and since $\langle y\rangle=y$ and $\|y\|=|y|$ near 0 , it suffices to prove that

$$
|p(x, y)| \gtrsim_{C_{0}, C_{1}, C_{2}}\left|\left(x-\gamma_{1}^{j}\right)+t\left(y-\gamma_{2}^{j}\right)\right|,
$$

and

$$
|p(x, y)| \gtrsim_{C_{0}, C_{1}, C_{2}}\left|x-\gamma_{1}^{j}\right|^{2}+\left|y-\gamma_{2}^{j}\right|^{2},
$$

for $(x, y)$ in a sufficiently small neighborhood (on $\left.\mathbb{R}^{2}\right)$ of $\left(\gamma_{1}^{j}, \gamma_{2}^{j}\right)$.

We distinguish two cases. The non-degenerate case is when $\frac{C_{1} e\left(\gamma_{1}^{j}\right)}{C_{2} e\left(\gamma_{2}^{j}\right)}$ is not a real number. This is the same as saying that $\left|C_{0}\right|,\left|C_{1}\right|,\left|C_{2}\right|$ form a non-degenerate triangle. By using a Taylor expansion we get

$$
\frac{p(x, y)}{2 \pi i}=
$$

$C_{1} e\left(\gamma_{1}^{j}\right)\left(x-\gamma_{1}^{j}+\pi i\left(x-\gamma_{1}^{j}\right)^{2}+O\left(\left|x-\gamma_{1}^{j}\right|^{3}\right)\right)+C_{2} e\left(\gamma_{2}^{j}\right)\left(\left(y-\gamma_{2}^{j}\right)+\pi i\left(y-\gamma_{2}^{j}\right)^{2}+O\left(\left|y-\gamma_{2}^{j}\right|^{3}\right)\right)$.

Note that

$$
\left|C_{1} e\left(\gamma_{1}^{j}\right)\left(x-\gamma_{1}^{j}\right)+C_{2} e\left(\gamma_{2}^{j}\right)\left(y-\gamma_{2}^{j}\right)\right| \gtrsim_{C_{0}, C_{1}, C_{2}}\left(\left(x-\gamma_{1}^{j}\right)^{2}+\left(y-\gamma_{2}^{j}\right)^{2}\right)^{1 / 2},
$$

and thus $|p(x, y)| \gtrsim_{C_{0}, C_{1}, C_{2}}\left(\left(x-\gamma_{1}^{j}\right)^{2}+\left(y-\gamma_{2}^{j}\right)^{2}\right)^{1 / 2} \geq\left(x-\gamma_{1}^{j}\right)^{2}+\left(y-\gamma_{2}^{j}\right)^{2}$, for $\left|x-\gamma_{1}^{j}\right|,\left|y-\gamma_{2}^{j}\right|$ sufficiently small. Hence (1) also holds with, say, $t=1$.

The degenerate case is when $\left|C_{0}\right|,\left|C_{1}\right|,\left|C_{2}\right|$ form a degenerate triangle. In this case there is only one zero, call it $\left(\gamma_{1}, \gamma_{2}\right)$. It follows that $C_{0}, C_{1} e\left(\gamma_{1}\right), C_{2} e\left(\gamma_{2}\right)$ are real multiples of each other. Thus, there must exist two among these three numbers with a positive ratio. There are two cases.

First let us assume $t:=\frac{C_{2} e\left(\gamma_{2}\right)}{C_{1} e\left(\gamma_{1}\right)}>0$. Then, proceeding as before,

$$
\begin{gathered}
\frac{p(x, y)}{2 \pi i C_{1} e\left(\gamma_{1}\right)}=\left(x-\gamma_{1}+t\left(y-\gamma_{2}\right)+O\left(\left|x-\gamma_{1}\right|^{3}+\left|y-\gamma_{2}\right|^{3}\right)\right)+ \\
\pi i\left(\left(x-\gamma_{1}\right)^{2}+t\left(y-\gamma_{2}\right)^{2}+O\left(\left|x-\gamma_{1}^{j}\right|^{3}+\left|y-\gamma_{2}\right|^{3}\right)\right)
\end{gathered}
$$

where the first term is the real part, while the second term is the imaginary part. Since $t>0$,

$$
\left(x-\gamma_{1}\right)^{2}+t\left(y-\gamma_{2}\right)^{2} \gtrsim_{t}\left(x-\gamma_{1}\right)^{2}+\left(y-\gamma_{2}\right)^{2}>>\left(\left|x-\gamma_{1}\right|^{3}+\left|y-\gamma_{2}\right|^{3}\right),
$$

for $\left|x-\gamma_{1}\right|,\left|y-\gamma_{2}\right|$ sufficiently small. If $\left|\left(x-\gamma_{1}\right)+t\left(y-\gamma_{2}\right)\right| \geq\left(x-\gamma_{1}\right)^{2}+\left(y-\gamma_{2}\right)^{2}$, then the real part is dominant, and thus $|p(x, y)| \gtrsim\left|\left(x-\gamma_{1}\right)+t\left(y-\gamma_{2}\right)\right|$. If $\mid(x-$ $\left.\gamma_{1}\right)+t\left(y-\gamma_{2}\right) \mid \leq\left(x-\gamma_{1}\right)^{2}+\left(y-\gamma_{2}\right)^{2}$, then the imaginary part is dominant, and thus $|p(x, y)| \gtrsim\left(x-\gamma_{1}\right)^{2}+\left(y-\gamma_{2}\right)^{2}$.

The second possibility is that $s:=\frac{C_{1} e\left(\gamma_{1}\right)}{C_{0}}>0$. The case $\frac{C_{2} e\left(\gamma_{2}\right)}{C_{0}}>0$ is completely symmetric, so we omit it. Note that

$$
\begin{gathered}
|p(x, y)|=\left|C_{2}+C_{0} e\left(-\gamma_{2}\right) e\left(\gamma_{2}-y\right)+C_{1} e\left(\gamma_{1}-\gamma_{2}\right) e\left(x-\gamma_{1}-\left(y-\gamma_{2}\right)\right)\right|= \\
2 \pi \mid C_{0} e\left(-\gamma_{2}\right)\left(\left(\gamma_{2}-y\right)+\pi i\left(\gamma_{2}-y\right)^{2}+O\left(\left|\gamma_{2}-y\right|^{3}\right)\right)+ \\
C_{1} e\left(\gamma_{1}-\gamma_{2}\right)\left(\left(x-\gamma_{1}-\left(y-\gamma_{2}\right)\right)+\pi i\left(x-\gamma_{1}-\left(y-\gamma_{2}\right)\right)^{2}+O\left(\left|x-\gamma_{1}-\left(y-\gamma_{2}\right)\right|^{3}\right)\right) \mid
\end{gathered}
$$

Thus,

$$
\frac{|p(x, y)|}{\left|2 \pi C_{0} e\left(-\gamma_{2}\right)\right|}=
$$




$$
\begin{gathered}
\mid\left(\left(\gamma_{2}-y\right)+s\left(x-\gamma_{1}-\left(y-\gamma_{2}\right)\right)+O_{s}\left(\left|\gamma_{2}-y\right|^{3}+\left|x-\gamma_{1}\right|^{3}\right)\right)+ \\
\pi i\left(\left(\gamma_{2}-y\right)^{2}+s\left(x-\gamma_{1}-\left(y-\gamma_{2}\right)\right)^{2}+O_{s}\left(\left|\gamma_{2}-y\right|^{3}+\left|x-\gamma_{1}\right|^{3}\right)\right) \mid .
\end{gathered}
$$

Using the fact that

$\left(\gamma_{2}-y\right)^{2}+s\left(x-\gamma_{1}-\left(y-\gamma_{2}\right)\right)^{2} \gtrsim_{s}\left(\gamma_{2}-y\right)^{2}+\left(x-\gamma_{1}-\left(y-\gamma_{2}\right)\right)^{2} \geq \frac{\left(\gamma_{2}-y\right)^{2}+\left(x-\gamma_{1}\right)^{2}}{4}$,

(1) and (2) follow as before, this time with $t:=-\frac{1+s}{s}$.

We will for the rest of the paper implicitly assume $\beta>0$. The following result uses simultaneous diophantine approximation to construct sharp almost periods. The requirement (i) will be needed later, in order to be able to place the generators $x$ and $x+\left\{P_{k}\right\}$ of the two orbits, in the (potentially very small) interval $I$ where the "solution" $f$ is guaranteed to be nonzero.

Lemma 2.2. Let $\alpha, \beta \in \mathbb{R}$ be two nonzero numbers with $\alpha / \beta$ irrational. Let also $1>s>0$ be fixed. Then there exists a constant $0<D=D(s, \alpha, \beta)<\infty$ and a sequence $N_{k}$ of positive integers going to infinity such that for each $k \geq 1$

(i) $\left\{\frac{N_{k}}{\beta}\right\}<s$,

(ii) $N_{k}\left\|N_{k} \frac{\alpha}{\beta}\right\| \leq D \min _{1 \leq n \leq N_{k}} n\left\|n \frac{\alpha}{\beta}\right\|$,

(iii) $N_{k}\left\|N_{k} \frac{\alpha}{\beta}\right\| \leq D$

Proof. We have two cases. If (call this the badly approximable regime)

$$
\liminf _{N \rightarrow \infty} N\left\|N \frac{\alpha}{\beta}\right\|>0
$$

then let $\epsilon:=\min _{N \in \mathbb{N}} N\left\|N \frac{\alpha}{\beta}\right\|>0$. Dirichlet's Theorem implies that we can choose a sequence $N_{k}^{\prime} \rightarrow \infty$ such that $N_{k}^{\prime}\left\|N_{k}^{\prime} \frac{\alpha}{\beta}\right\| \leq 1$ for each $k$. But then

$$
N_{k}^{\prime}\left\|N_{k}^{\prime} \frac{\alpha}{\beta}\right\| \leq \frac{1}{\epsilon} \min _{n \in \mathbb{N}} n\left\|n \frac{\alpha}{\beta}\right\| .
$$

Finally, by pigeonholing, for each $k$ there must exist some $1 \leq m_{k} \leq 1 / s$ such that $N_{k}:=m_{k} N_{k}^{\prime}$ satisfies (i). It is immediate that

$$
N_{k}\left\|N_{k} \frac{\alpha}{\beta}\right\| \leq \frac{1}{s^{2} \epsilon} \min _{n \in \mathbb{N}} n\left\|n \frac{\alpha}{\beta}\right\| .
$$

Assume next that we are in the well approximable regime, that is

$$
\liminf _{N \rightarrow \infty} N\left\|N \frac{\alpha}{\beta}\right\|=0 .
$$

This is equivalent with saying the the sequence $a_{k}$ in the continued fraction of $\alpha / \beta$ is unbounded. Let $\left(p_{k}, N_{k}^{\prime}\right)$ be the sequence of best approximants for $\frac{\alpha}{\beta}$, ordered such that $N_{k}^{\prime}$ is increasing. Thus

$$
\left|\frac{\alpha}{\beta}-\frac{p_{k}}{N_{k}^{\prime}}\right| \leq \frac{1}{N_{k}^{\prime} N_{k+1}^{\prime}} .
$$

Recall that $N_{k+1}^{\prime} \geq a_{k} N_{k}^{\prime}$, and thus $s N_{k+1}^{\prime}>N_{k}^{\prime}$ for each $k \in E$, where $E$ is infinite. It is known (see for example Theorem 7.13 in [6]) that for each $k$

$$
N_{k}^{\prime}\left\|N_{k}^{\prime} \frac{\alpha}{\beta}\right\|=\min _{1 \leq n<N_{k+1}^{\prime}} n\left\|n \frac{\alpha}{\beta}\right\| .
$$


Let $\pi: \mathbb{N} \rightarrow E$ be an increasing bijection. Choose as before $1 \leq m_{k} \leq 1 / s$ such that $N_{k}:=m_{k} N_{\pi(k)}^{\prime}$ satisfies (i). Note that $N_{k}<N_{\pi(k)+1}^{\prime}$, and thus (ii)-(iii) follow as before.

The next lemma will be needed in the proof of Proposition 2.4.

Lemma 2.3. Let $C_{0}, C_{1}, C_{2} \in \mathbb{C}$ be some nonzero complex numbers. Let $\alpha, \beta$ be some nonzero real numbers. Define

$$
P(x)=C_{0}+C_{1} e(\alpha x)+C_{2} e(\beta x) .
$$

Let $\left(N_{k}\right)$ be a sequence such that (ii) and (iii) in Lemma 2.2 hold. Define $\frac{1}{M_{k}}:=$ $\frac{N_{k}\left\|N_{k} \frac{\alpha}{\beta}\right\|}{D}$, and let $P_{k}:=\frac{N_{k}}{\beta}$. Then for each $k$ and each $\delta>0$, there exists an exceptional set $E_{k, \delta} \subset[0,1]$ such that

$$
\left|E_{k, \delta}\right|<\delta
$$

and

$$
\frac{1}{M_{k} P_{k}} \sum_{n=0}^{\left[P_{k}\right]-1} \frac{1}{|P(x+n)|} \lesssim_{\delta, C_{0}, C_{1}, C_{2}, \alpha, \beta} \log P_{k},
$$

for each $x \in[0,1] \backslash E_{k, \delta}$.

Proof. Let $\left(\gamma_{1}, \gamma_{2}\right) \in[0,1]^{2}$ be a real zero of the polynomial $p(x, y)=C_{0}+C_{1} e(x)+$ $\mathrm{C}_{2} e(y)$. Define

$$
A_{n}(x):=\left\|\alpha(x+n)-\gamma_{1}\right\|^{2}+\left\|\beta(x+n)-\gamma_{2}\right\|^{2}
$$

By Lemma 2.1, it suffices to find an exceptional set with $\left|E_{k, \delta}\right| \leq \frac{\delta}{2}$, such that

$$
\frac{1}{P_{k} M_{k}} \sum_{n=0}^{\left[P_{k}\right]-1} \frac{1}{A_{n}(x)} \lesssim_{\delta, \alpha, \beta} \log P_{k},
$$

for each $x \in[0,1] \backslash E_{k, \delta}$. The heuristics behind the proof is that if $\alpha / \beta$ is less Diophantine, then the estimate above holds because $M_{k}$ is large, while if $\alpha / \beta$ is Diophantine, we win because of extra regularity of the counting measure for the points $(n \alpha, n \beta)$.

The key observation is that each strip

$$
S_{a}:=\left\{(x, y): a-\frac{1}{4 M_{k} P_{k}} \leq \beta x-\alpha y<a+\frac{1}{4 M_{k} P_{k}}\right\}
$$

contains at most $O_{\beta}(1)$ points $\left(\left\{-n \alpha+\gamma_{1}\right\},\left\{-n \beta+\gamma_{2}\right\}\right), 0 \leq n \leq\left[P_{k}\right]-1$. Indeed, assume for contradiction that some $S_{a}$ contains both $\left(\left\{-n \alpha+\gamma_{1}\right\},\left\{-n \beta+\gamma_{2}\right\}\right)$ and $\left(\left\{-m \alpha+\gamma_{1}\right\},\left\{-m \beta+\gamma_{2}\right\}\right)$, with $\left[P_{k}\right]-\frac{1}{\beta}>|m-n|>\frac{2}{\beta}$. It follows that

$$
\left|\beta\left(\left[-n \alpha+\gamma_{1}\right]-\left[-m \alpha+\gamma_{1}\right]\right)-\alpha\left(\left[-n \beta+\gamma_{2}\right]-\left[-m \beta+\gamma_{2}\right]\right)\right|<\frac{1}{M_{k} P_{k}} .
$$

Note that $L:=\left|\left[-n \beta+\gamma_{2}\right]-\left[-m \beta+\gamma_{2}\right]\right| \neq 0$ and $L \leq|-n \beta+m \beta|+1 \leq N_{k}$. The above implies that $\left\|L \frac{\alpha}{\beta}\right\|<\frac{1}{M_{k} P_{k} \beta} \leq \frac{1}{M_{k} L}$, which contradicts (ii) in Lemma 2.2 .

Let $\mathcal{C}$ be the collection of all points $\left\{-n \alpha+\gamma_{1}\right\}$ with $0 \leq n \leq\left[P_{k}\right]-1$. Let $E_{\delta}^{\prime}$ be the set of $x \in[0,1)$ such that the distance from $\{\alpha x\}$ to $\mathcal{C}$ is less than $\frac{\delta|\alpha|}{20 P_{k}}$. Then $\left|E_{\delta}^{\prime}\right|<\delta / 10$. 
Let $E_{\delta}^{\prime \prime}$ consist of those $x \in[0,1)$ with $\min (\|\alpha x\|,\|\beta x\|) \leq \frac{\delta(|\alpha|+|\beta|)}{100}$. Then $\left|E_{\delta}^{\prime \prime}\right| \leq$ $\frac{\delta}{10}$.

Note that if $u, v \in[0,1)$ with $\|u\|>\epsilon$ for some $\epsilon>0$, then

$$
\|u-v\| \gtrsim_{\epsilon}|u-v| \text {. }
$$

Split $[0,1) \backslash E_{\delta}^{\prime \prime}$ in intervals $H \in \mathcal{H}$, such that for each $H$, the integer parts $[\alpha x]$ and $[\beta x]$ are both constant, when $x$ varies through $H$. Thus, the points $(\{\alpha x\},\{\beta x\})$, $x \in H$ sit on a fixed line $\beta x-\alpha y=c_{H}$. Note that $\mathcal{H}$ contains $O_{\alpha, \beta}(1)$ intervals.

Let $H \in \mathcal{H}$. Then, using (4),

$$
\begin{gathered}
\int_{H \backslash E_{\delta^{\prime}}} \frac{1}{P_{k} M_{k}} \sum_{n=0}^{\left[P_{k}\right]-1} \frac{1}{A_{n}(x)} d \lesssim \alpha, \beta, \delta \\
\int_{H \backslash E_{\delta^{\prime}}} \frac{1}{P_{k} M_{k}} \sum_{n=0}^{\left[P_{k}\right]-1} \frac{1}{\left|\{\alpha x\}-\left\{-n \alpha+\gamma_{1}\right\}\right|^{2}+\left|\{\beta x\}-\left\{-n \beta+\gamma_{2}\right\}\right|^{2}} d x .
\end{gathered}
$$

The key observation implies that there are $O_{\beta}(1)$ points $\left(\left\{-n \alpha+\gamma_{1}\right\},\left\{-n \beta+\gamma_{2}\right\}\right)$ in each strip $S t_{j}:=\left\{(x, y): c_{H}+\frac{j}{2 M_{k} P_{k}} \leq \beta x-\alpha y<c_{H}+\frac{j+1}{2 M_{k} P_{k}}\right\}$, with, say, $-10 M_{k} P_{k} \leq j \leq 10 M_{k} P_{k}$. Call $B_{j}$ the set of $n$ corresponding to these points. We first evaluate for $j \notin\{-1,0\}$,

$$
\int_{H} \sum_{\substack{n=0 \\ n \in B_{j}}}^{\left[P_{k}\right]-1} \frac{1}{\left|\{\alpha x\}-\left\{-n \alpha+\gamma_{1}\right\}\right|^{2}+\left|\{\beta x\}-\left\{-n \beta+\gamma_{2}\right\}\right|^{2}} d x .
$$

Since $(\{\alpha x\},\{\beta x\})_{x \in H}$ belong to a line segment, and since the points in $S t_{j}$ belong to a strip parallel to and at distance at least $|j| / 2 M_{k} P_{k}$ from this line segment, a simple change of coordinates shows that the integral above is dominated by $O_{\alpha, \beta}\left(\int_{0}^{1} \frac{1}{x^{2}+\left(\frac{|j|}{M_{k} P_{k}}\right)^{2}} d x\right)=O_{\alpha, \beta}\left(\frac{M_{k} P_{k}}{|j|}\right)$. Of course, only at most $P_{k}$ values of $j$ can contribute, and thus

$$
\begin{aligned}
\int_{H} \frac{1}{M_{k} P_{k}} \sum_{\substack{n=0 \\
n \in \cup_{j \notin\{0,1\}} B_{j}}}^{\left[P_{k}\right]-1} \frac{1}{\left|\{\alpha x\}-\left\{-n \alpha+\gamma_{1}\right\}\right|^{2}+\left|\{\beta x\}-\left\{-n \beta+\gamma_{2}\right\}\right|^{2}} d \lesssim \alpha, \beta \\
\frac{1}{M_{k} P_{k}} \sum_{\substack{j \notin\{0,1\} \\
S_{j} \neq \emptyset}} \frac{M_{k} P_{k}}{|j|} \lesssim \log P_{k} .
\end{aligned}
$$

On the other hand, if $n \in B_{0} \cup B_{-1}$, then we use the fact that if $x \notin E_{\delta}^{\prime}$ then

$$
\left|\{\alpha x\}-\left\{-n \alpha+\gamma_{1}\right\}\right|^{2}+\left|\{\beta x\}-\left\{-n \beta+\gamma_{2}\right\}\right|^{2} \gtrsim \frac{\delta^{2}|\alpha|^{2}}{P_{k}^{2}} .
$$

Using this, the fact that $B_{0} \cup B_{-1}$ contains $O_{\beta}(1)$ points and the fact that $M_{k} \geq 1$, we get via a change of variables that

$$
\int_{H \backslash E_{\delta}^{\prime}} \frac{1}{M_{k} P_{k}} \sum_{\substack{n=0 \\ n \in B_{0} \cup B_{-}}}^{\left[P_{k}\right]-1} \frac{1}{\left|\{\alpha x\}-\left\{-n \alpha+\gamma_{1}\right\}\right|^{2}+\left|\{\beta x\}-\left\{-n \beta+\gamma_{2}\right\}\right|^{2}} d x
$$


is dominated by $\left.\frac{1}{M_{k} P_{k}} O\left(\int_{\frac{\delta^{2}|\alpha|^{2}}{P_{k}^{2}}}^{1} \frac{1}{x^{2}} d x\right)\right)=O_{\alpha, \beta}\left(\frac{P_{k}}{\delta M_{k} P_{k}}\right)=O_{\alpha, \beta, \delta}(1)$. Thus

$$
\begin{gathered}
\int_{[0,1) \backslash\left(E_{\delta^{\prime}} \cup E_{\delta}^{\prime \prime}\right)} \frac{1}{M_{k} P_{k}} \sum_{n=0}^{\left[P_{k}\right]-1} \frac{1}{\left|\{\alpha x\}-\left\{-n \alpha+\gamma_{1}\right\}\right|^{2}+\left|\{\beta x\}-\left\{-n \beta+\gamma_{2}\right\}\right|^{2}} d x= \\
\sum_{H \in \mathcal{H}} \int_{H \backslash E_{\delta^{\prime}}} \frac{1}{M_{k} P_{k}} \sum_{n=0}^{\left[P_{k}\right]-1} \frac{1}{\left|\{\alpha x\}-\left\{-n \alpha+\gamma_{1}\right\}\right|^{2}+\left|\{\beta x\}-\left\{-n \beta+\gamma_{2}\right\}\right|^{2}} d x \\
\lesssim_{\alpha, \beta, \delta} \log P_{k},
\end{gathered}
$$

since there are $O_{\alpha, \beta}(1)$ intervals $H$. It follows that

$$
\left|E_{\delta}^{\prime \prime \prime}:=\left\{x \in[0,1) \backslash\left(E_{\delta^{\prime}} \cup E_{\delta}^{\prime \prime}\right): \frac{1}{M_{k} P_{k}} \sum_{n=0}^{\left[P_{k}\right]-1} \frac{1}{|P(x+n)|} \geq C_{\alpha, \beta, \delta} \log P_{k}\right\}\right| \leq \delta / 10,
$$

if $C_{\alpha, \beta, \delta}$ is chosen large enough. Finally, define $E_{k, \delta}:=E_{\delta}^{\prime} \cup E_{\delta}^{\prime \prime} \cup E_{\delta}^{\prime \prime \prime}$.

The next proposition captures the almost orthogonality phenomenon. The idea is to avoid estimating products along individual orbits (which seems nearly impossible), but rather to compare products along two orbits.

Proposition 2.4. Let $\left(N_{k}\right)$ be a sequence such that (ii) and (iii) in Lemma 2.2 hold, and define $P_{k}:=\frac{N_{k}}{\beta}$. Let $C_{0}, C_{1}, C_{2} \in \mathbb{C}$ and $\alpha, \beta \in \mathbb{R}$ be nonzero and define $P(x)=C_{0}+C_{1} e(\alpha x)+C_{2} e(\beta x)$. Given $\delta>0$, there exists $E_{k, \delta} \subset[0,1]$ such that $\left|E_{k, \delta}\right| \leq \delta$, and there exists a universal constant $L=L\left(\delta, C_{0}, C_{1}, C_{2}, \alpha, \beta\right)$ such that for each $x, y$ satisfying $x \in[0,1) \backslash E_{k, \delta}$ and $x=y+P_{k}$, we have

$$
\left|\prod_{n=0}^{\left[P_{k}\right]-1} P(y+n)\right| \leq P_{k}^{L}\left|\prod_{n=0}^{\left[P_{k}\right]-1} P(x+n)\right| .
$$

Proof. Let as before

$$
\frac{1}{M_{k}}:=\frac{P_{k}\left\|P_{k} \frac{\alpha}{\beta}\right\|}{D}<1
$$

Then, we have

$|e(\alpha x)-e(\alpha y)|=\left|e\left(P_{k} \alpha\right)-1\right| \leq \frac{10 D}{P_{k} M_{k}} \quad$ and $\quad|e(\beta x)-e(\beta y)|=\left|e\left(P_{k} \beta\right)-1\right| \leq \frac{10 D}{P_{k} M_{k}}$.

Thus, for each $n \in \mathbb{N}$

$$
|P(y+n)| \leq|P(x+n)|+\frac{C D}{M_{k} P_{k}}
$$

for some universal constant $C:=100\left(\left|C_{1}\right|+\left|C_{2}\right|\right)$.

Use the fact $a+b \leq a e^{\frac{b}{a}}$ for each $a, b>0$ to get

$$
|P(y+n)| \leq|P(x+n)| e^{\frac{C D}{M_{k} P_{k}|P(x+n)|}}
$$

and thus

$$
\left|\prod_{n=0}^{\left[P_{k}\right]-1} P(y+n)\right| \leq\left|\prod_{n=0}^{\left[P_{k}\right]-1} P(x+n)\right| e^{\frac{C D}{M_{k} P_{k}} \sum_{n=0}^{\left[P_{k}\right]-1} \frac{1}{|P(x+n)|} .}
$$

The result now follows from Lemma 2.3. 
Proof. (of Theorem 1.3 for $(1,3)$ configurations)

It was proved in [4] that the linear independence of the time-frequency translates is preserved under area preserving affine transformations of the plane, both for $L^{2}$ and for Schwartz functions. Thus, it is easy to see that any $(1,3)$ configuration can be reduced to a special $(1,3)$ configuration like $(0,0),(1,0),(1, \alpha),(1, \beta)$, for some nonzero $\alpha, \beta$, with $\alpha \neq \beta$. Indeed, apply first a rotation, then a joint rescaling of the time and frequency axes so that the distance between the line containing the 3 points and the remaining point becomes 1 , followed by a translate along the frequency axis and then by a vertical shear. It is also clear that it is precisely the $(1,3)$ configurations (and they only), that can be reduced to the special configuration from above. This follows since any linear transformation of the plane maps collinear points into collinear points.

We can also assume that the 4 points do not sit on a lattice, since that case is covered by the work in [5]. In particular, we assume $\alpha / \beta$ is irrational.

Assume now for contradiction that there is some nontrivial Schwartz $f: \mathbb{R} \rightarrow \mathbb{C}$ such that for a.e. $x$, for some fixed nonzero constants $C_{i} \in \mathbb{C}$

$$
f(x+1)=f(x)\left(C_{0}+C_{1} e(\alpha x)+C_{2} e(\beta x)\right) .
$$

We denote as before $P(x)=C_{0}+C_{1} e(\alpha x)+C_{2} e(\beta x)$.

Let $(I, S, d)$ be a triple such that $d>0, I \subset[0,1)$ is an interval, $S \subset I$ is a set with full Lebesgue measure $|I|$, such that

$$
\begin{gathered}
\lim _{\substack{|n| \rightarrow \infty \\
n \in \mathbb{Z}}}|n|^{C} f(x+n)=0, \text { for each } x \in S \text { and each } C>0, \\
d<|f(x)|<d^{-1}, \text { for each } x \in S \cup S+1, \\
f(x+1)=f(x)\left(C_{0}+C_{1} e(\alpha x)+C_{2} e(\beta x)\right), \text { for each } x \in S+\mathbb{Z} .
\end{gathered}
$$

We will refer to $C_{0}, C_{1}, C_{2}, I, S, d, \alpha, \beta$ as fundamental parameters. Implicit constants in inequalities involving $\lesssim$ are allowed to depend on these parameters, since they can be thought of as being fixed for the rest of the argument.

Apply Lemma 2.2 with $s:=|I| / 10$, to get a constant $D=D(|I|, \alpha, \beta)>0$ and a sequence $N_{k}$ of positive integers going to infinity such that for each $k \geq 1$

(i) $\left\{P_{k}:=\frac{N_{k}}{\beta}\right\}<|I| / 10$,

(ii) $N_{k}\left\|N_{k} \frac{\alpha}{\beta}\right\| \leq D \min _{1 \leq n \leq N_{k}} n\left\|n \frac{\alpha}{\beta}\right\|$,

(iii) $N_{k}\left\|N_{k} \frac{\alpha}{\beta}\right\| \leq D$

By Proposition 2.4 with $\delta=\frac{|I|}{100}$, for each $x \in[0,1) \backslash E_{k, \delta}$ and $x=y+P_{k}$ we have

$$
\left|\prod_{n=0}^{\left[P_{k}\right]-1} P(y+n)\right| \leq P_{k}^{L}\left|\prod_{n=0}^{\left[P_{k}\right]-1} P(x+n)\right| .
$$

Note that $\left(S \backslash E_{k, \delta}\right) \cap\left(\left\{P_{k}\right\}+S\right) \neq \emptyset$, since the intersection of each of the 2 sets with $I$ is large. Pick any $x_{k} \in S \backslash E_{k, \delta}$, such that also $x_{k}-\left\{P_{k}\right\} \in S$. Define $x_{k}^{\prime}:=x_{k}-\left\{P_{k}\right\}$. We will now argue that (5) can not hold for both $x_{k}$ (with $n \rightarrow \infty$ ) and $x_{k}^{\prime}$ (with $n \rightarrow-\infty)$. Let $y_{k}=x_{k}^{\prime}-\left[P_{k}\right]=x_{k}-P_{k}$. Note that

$$
\prod_{n=0}^{\left[P_{k}\right]-1} P\left(y_{k}+n\right)=\prod_{n=1}^{\left[P_{k}\right]} P\left(x_{k}^{\prime}-n\right)
$$


and thus, by (8) we get

$$
\left|\prod_{n=1}^{\left[P_{k}\right]} P\left(x_{k}^{\prime}-n\right)\right| \leq P_{k}^{L}\left|\prod_{n=0}^{\left[P_{k}\right]-1} P\left(x_{k}+n\right)\right| .
$$

Recall that we have

$$
\begin{gathered}
f\left(x_{k}+\left[P_{k}\right]\right)=f\left(x_{k}\right) \prod_{n=0}^{\left[P_{k}\right]-1} P\left(x_{k}+n\right) \\
f\left(x_{k}^{\prime}-\left[P_{k}\right]\right)=f\left(x_{k}^{\prime}\right)\left[\prod_{n=1}^{\left[P_{k}\right]} P\left(x_{k}^{\prime}-n\right)\right]^{-1} .
\end{gathered}
$$

Using (6) and (9), we get that

$$
\left|f\left(x_{k}+\left[P_{k}\right]\right) f\left(x_{k}^{\prime}-\left[P_{k}\right]\right)\right| \gtrsim P_{k}^{-L},
$$

with an implicit constant depending only on the fundamental parameters (in particular, independent of $k$ ). This clearly contradicts (5), if we let $k \rightarrow \infty$.

\section{Proof of Theorem 1.3 for $(2,2)$ configurations}

The reader can easily check that the argument for $(1,3)$ configurations presented above also works here. In particular, the existence of large $P \in \mathbb{R}$ satisfying

$$
\max \{\|P \alpha\|,\|P \beta\|\} \lesssim 1 / P
$$

implies that one can run the almost periodicity argument.

We also present an alternative, simpler argument, which does not work in the $(1,3)$ case. As before, by using metaplectic transformations we can reduce to the case of special $(2,2)$ configurations. Assume there is a continuous function $f: \mathbb{R} \rightarrow \mathbb{C}$ such that $f$ is nonzero on some interval $I \in[0,1$ ), satisfying a weaker assumption (minimal decay)

$$
\lim _{\substack{|n| \rightarrow \infty \\ n \in \mathbb{Z}}} f(x+n)=0
$$

for a.e. $x \in[0,1]$, and

$$
f(x+1)(A+B e(\alpha x))=f(x)(C+D e(\beta x)),
$$

for a.e. $x$, for some fixed $A, B, C, D \in \mathbb{C}, \alpha, \beta \in \mathbb{R}$, none of them zero. We can also assume that $\alpha$ and $\beta$ are rationally independent, by otherwise invoking the lattice case. Note that we assume far less, namely minimal (rather than Schwartz) decay. Thus, the result we prove for special $(2,2)$ configurations is in some sense best possible.

First, let us deal with the case when both $\alpha, \beta$ are irrational. We can trivially assume $A$ is real. Let

$$
P(x)=A+B e(\alpha x) \text { and } Q(x)=C+D e(\beta x) .
$$

We first observe that $|A| \neq|B|$. Indeed, assume for contradiction that $|A|=|B|$. Then, there exists $\omega \in[0,1)$ such that $P\left(\omega+n \alpha^{-1}\right)=0$ for each $n \in \mathbb{Z}$. There exist infinitely many $n$ such that $x_{n}:=\left\{\omega+n \alpha^{-1}\right\} \in I$. Choose such an $n_{0} \in \mathbb{N}$ with the additional property that $Q\left(\omega+n \alpha^{-1}+m\right) \neq 0$ when $n=n_{0}$, for each $m \in \mathbb{Z}$ (it is 
easy to check, using the fact that $\alpha$ and $\beta$ are rationally independent, that this holds for all $n \in \mathbb{Z}$ with at most one possible exception). It follows that there exists an interval $H$ around $x_{n_{0}}$ such that $\inf _{0 \leq n \leq\left[\omega+n_{0} \alpha^{-1}\right], x \in H}|Q(x+n)|>0$.

Now, by using the recurrence (14) along the orbits $x, x+1, \ldots, x+\left[\omega+n_{0} \alpha^{-1}\right]-1$ of points $x \in I \cap H$, we get that $\sup _{y \in\left[\omega+n_{0} \alpha^{-1}\right]+H}|f(y)|=\infty$, contradicting the continuity of $f$. Thus, we have proved that $\inf _{x \in \mathbb{R}}|P(x)|>0$. Similarly, it follows that $|C| \neq|D|$, and thus $\inf _{x \in \mathbb{R}}|Q(x)|>0$.

This is the only place in the argument where the continuity of $f$ is exploited. Continuity seems to be vital in order to rule out the real zeros for $P$ and $Q$. Indeed, if $f$ is not continuous, the set $\{x \in[0,1]: 0<|f(x)|\}$, while it has positive measure, is not guaranteed to have among its accumulation points any of the zeros of $P$ and $Q$.

The functions $\psi(x)=\ln |A+B e(x)|$ and $\phi(x)=\ln |C+D e(x)|$ are now guaranteed to be continuous on $[0,1)$. Let $S \subset I$ be a set with Lebesgue measure $|I|$ such that (13) and (14) hold for each $x \in S$. Due to (13), it follows that for each $x, z \in S$

$$
\lim _{N \rightarrow \infty}\left(\sum_{n=1}^{N} \phi(\beta x+\beta n)-\sum_{n=1}^{N} \psi(\alpha x+\alpha n)\right)=-\infty
$$

and

$$
\lim _{N \rightarrow \infty}\left(\sum_{n=-N}^{-1} \phi(\beta z+\beta n)-\sum_{n=-N}^{-1} \psi(\alpha z+\alpha n)\right)=+\infty
$$

Let $p_{k}, q_{k}$ relatively prime, $q_{k} \rightarrow \infty$ such that

$$
\left|\beta-\frac{p_{k}}{q_{k}}\right| \leq \frac{1}{q_{k}^{2}}
$$

Note that $n \beta$ is very regular, that is

$$
\left|n \beta-\frac{n p_{k}}{q_{k}}\right| \leq \frac{1}{q_{k}}, \quad-q_{k} \leq n \leq q_{k},
$$

where $\left(n p_{k} \bmod q_{k}\right)_{n=1}^{q_{k}}$ cover all the residues $\bmod q_{k}$. Using this and $\left|\phi^{\prime}\right| \gtrsim 1$, we get

$$
\left|\sum_{n=1}^{q_{k}} \phi(\beta y+\beta n)-q_{k} \int_{0}^{1} \phi\right|=O(1)
$$

and

$$
\left|\sum_{n=-q_{k}}^{-1} \phi(\beta y+\beta n)-q_{k} \int_{0}^{1} \phi\right|=O(1)
$$

for each $y \in[0,1]$ (use Riemann sums). An immediate corollary is that for each $x, z \in S$

$$
\left|\sum_{n=1}^{q_{k}} \phi(\beta x+\beta n)-\sum_{n=-q_{k}}^{-1} \phi(\beta z+\beta n)\right|=O(1)
$$

Let $B=r e(\theta)$, with $r>0$. By invoking Birkhoff's pointwise ergodic theorem for the function $1_{S}$, there exists $x \in S$ and some $n^{\prime} \in \mathbb{N}$ such that $z:=\left\{-x-\frac{2 \theta}{\alpha}+n^{\prime} \alpha^{-1}\right\} \in$ $S$. Let $y:=-x-\frac{2 \theta}{\alpha}+n^{\prime} \alpha^{-1}$ and $m=y-z$. 
The point of this selection is that for each $n, A+B e(\alpha y-n \alpha)$ and $A+B e(\alpha x+n \alpha)$ are complex conjugates and thus

$$
\sum_{n=-N+m}^{-1+m} \psi(\alpha z+\alpha n)=\sum_{n=1}^{N} \psi(\alpha x+\alpha n) .
$$

This implies that for each $N$,

$$
\left|\sum_{n=-N}^{-1} \psi(\alpha z+\alpha n)-\sum_{n=1}^{N} \psi(\alpha x+\alpha n)\right|=O(1) .
$$

But now it immediately follows that (15)-(18) cannot simultaneously hold.

If -say- $\alpha$ is irrational, apply the conjugates trick to $P$ as above, and use the periodicity of $Q$, like in the proof of Theorem 1.5 (b) described in Section 5 .

\section{Proof of Theorem 1.4}

Let us first prove part (a) of the theorem. Part (b) is proved in the end of this section. The hardest case is if $\frac{\alpha}{\beta}$ is Diophantine. This means that there exists $\gamma>1$ and there exists $\epsilon>0$ small enough to satisfy, say, $\frac{\epsilon}{\gamma+\epsilon-1} \leq \frac{1}{10}$, such that

$$
\liminf _{n \rightarrow \infty} n^{\gamma}\left\|n \frac{\alpha}{\beta}\right\|<\infty
$$

and in addition

$$
\inf _{n \in \mathbb{N}} n^{\gamma+\epsilon}\left\|n \frac{\alpha}{\beta}\right\|:=D_{\epsilon}>0 .
$$

The easier case is when $\frac{\alpha}{\beta}$ is Liouville, that is if for each $\eta>1$

$$
\liminf _{n \rightarrow \infty} n^{\eta}\left\|n \frac{\alpha}{\beta}\right\|<\infty .
$$

Lemma 4.1. Let $x_{1}, x_{2}, \ldots, x_{N}$ be $N$ not necessarily distinct real numbers. Then for each $N \in \mathbb{N}$ and each $\delta>0$, there exists an exceptional set $E_{N, \delta} \subset[0,1]$ such that

$$
\begin{gathered}
\left|E_{N, \delta}\right| \leq \delta, \\
\frac{1}{N} \sum_{n=1}^{N} \frac{1}{\left\|x-x_{n}\right\|} \lesssim_{\delta} \log N, \\
\frac{1}{N^{2}} \sum_{n=1}^{N} \frac{1}{\left\|x-x_{n}\right\|^{2}} \lesssim_{\delta} 1,
\end{gathered}
$$

for each $x \in[0,1] \backslash E_{N, \delta}$.

Proof. Since $y \mapsto\|y\|$ is 1 periodic, we can assume all $x_{i}$ are in $[0,1]$. Since $\left\|x-x_{i}\right\| \gtrsim$ $\min \left\{\left|x-x_{i}\right|,\left|x-\left(1-x_{i}\right)\right|\right\}$, by doubling the number of points if necessary, we can replace $\left\|x-x_{i}\right\|$ with $\left|x-x_{i}\right|$. Define

$$
U_{N, \delta}:=\bigcup_{i=1}^{N}\left[x_{i}-\frac{\delta}{10 N}, x_{i}+\frac{\delta}{10 N}\right],
$$


and note that

$$
\begin{gathered}
\int_{[0,1] \backslash U_{N, \delta}} \sum_{i=1}^{N} \frac{1}{\left|x-x_{i}\right|} d \lesssim N\left(\log N+\log \frac{1}{\delta}\right), \\
\int_{[0,1] \backslash U_{N, \delta}} \sum_{i=1}^{N} \frac{1}{\left|x-x_{i}\right|^{2}} d \lesssim N^{2} \delta^{-1} .
\end{gathered}
$$

The result now follows from Cebysev's inequality.

Lemma 4.2. Let $\alpha, \beta$ be some nonzero real numbers satisfying (20) above. Define also $M:=N^{\frac{(\gamma-1)(\gamma+\epsilon)}{\gamma+\epsilon-1}} D_{\epsilon}^{\frac{1}{\gamma+\epsilon-1}}$. Then for each $\xi \in \mathbb{R}$ and $N \in \mathbb{N}$

$$
\frac{1}{N^{\gamma}} \sum_{\substack{n=0 \\\left\|n \frac{\alpha}{\beta}-\xi\right\| \geq \frac{1}{M}}}^{N-1} \frac{1}{\left\|n \frac{\alpha}{\beta}-\xi\right\|} \lesssim_{\alpha, \beta, \gamma, \epsilon} 1 .
$$

Proof. The crucial observation is that for each $\frac{D_{\epsilon}}{2^{\gamma+\epsilon}} \geq R \geq \frac{1}{M}$, the $\|\cdot\|$-ball

$$
B(\xi, R):=\{x:\|x-\xi\|<R\}
$$

contains at most $N\left(\frac{R}{D}\right)^{\frac{1}{\gamma+\epsilon}}$ points $n \frac{\alpha}{\beta}$, with $0 \leq n \leq N-1$. Indeed, assume for contradiction that $N\left(\frac{R}{D}\right)^{\frac{1}{\gamma+\epsilon}}+1$ such points are contained in the ball. Then, two of these points would correspond to some $0 \leq n<m \leq N-1$ with $|n-m| \leq\left(\frac{D_{\epsilon}}{R}\right)^{\frac{1}{\gamma+\epsilon}}$. Thus,

$$
(m-n)^{\gamma+\epsilon}\left\|(m-n) \frac{\alpha}{\beta}\right\| \leq D_{\epsilon}
$$

which contradicts $(20)$.

In particular, for each $\frac{D_{\epsilon}}{2^{\gamma+\epsilon}} \geq 2^{-j} \geq \frac{1}{M}$ we have $O\left(N\left(\frac{1}{2^{j} D_{\epsilon}}\right)^{\frac{1}{\gamma+\epsilon}}\right)$ points in the ball $B_{j}:=B\left(\xi, 2^{-j}\right)$. Define for such a $j$

$$
S_{j}:=\left\{0 \leq n \leq N-1: n \frac{\alpha}{\beta} \in B_{j+1} \backslash B_{j}\right\} .
$$

Then, using the fact that there are at most $N$ points outside the ball $B\left(\xi\right.$, $\left.\frac{D_{\epsilon}}{2^{\gamma+\epsilon}}\right)$, we get

$$
\begin{gathered}
\frac{1}{N^{\gamma}} \sum_{\substack{n=0 \\
\left\|n \frac{\alpha}{\beta}-\xi\right\| \geq \frac{1}{M}}}^{N-1} \frac{1}{\left\|n \frac{\alpha}{\beta}-\xi\right\|} \leq \frac{1}{N^{\gamma}}\left(\sum_{\frac{D_{\epsilon}}{2^{\gamma+\epsilon}} \geq 2^{-j} \geq \frac{1}{M}} \sum_{n \in S_{j}} \frac{1}{\left\|n \frac{\alpha}{\beta}-\xi\right\|}+N \frac{2^{\gamma+\epsilon}}{D_{\epsilon}}\right) \\
\lesssim_{D_{\epsilon}, \gamma} \frac{1}{N^{\gamma}} \sum_{2^{-j} \geq \frac{1}{M}} 2^{j} N\left(\frac{1}{2^{j} D_{\epsilon}}\right)^{\frac{1}{\gamma+\epsilon} \lesssim \alpha, \beta, \gamma, \epsilon} 1 .
\end{gathered}
$$

We have the following analogue of Lemma 2.3

Lemma 4.3. Let $C_{0}, C_{1}, C_{2} \in \mathbb{C}$ and $\alpha, \beta$ be some nonzero numbers. Let $\gamma$ satisfy (20) if $\alpha / \beta$ is Diophantine and let $\gamma=2$ if $\alpha / \beta$ is Liouville. Define

$$
P(x)=C_{0}+C_{1} e(\alpha x)+C_{2} e(\beta x) .
$$


Then for each $N \in \mathbb{N}$ and $\delta>0$, there exists an exceptional set $E_{N, \delta} \subset[0,1]$ such that

$$
\left|E_{N, \delta}\right|<\delta
$$

and

$$
\frac{1}{N^{\gamma}} \sum_{n=0}^{N-1} \frac{1}{|P(x+n)|} \lesssim_{\delta, C_{0}, C_{1}, C_{2}, \alpha, \beta, \epsilon} 1,
$$

for each $x \in[0,1] \backslash E_{N, \delta}$.

Proof. Let $\left(\gamma_{1}, \gamma_{2}\right)$ be a zero of the polynomial $p(x, y)=C_{0}+C_{1} e(x)+C_{2} e(y)$, and let $t$ be the real number guaranteed by Lemma 2.1. Define

$$
A_{n}(x):=\left\|\alpha(x+n)-\gamma_{1}+t\left\langle\beta(x+n)-\gamma_{2}\right\rangle\right\|+\left\|\alpha(x+n)-\gamma_{1}\right\|^{2}+\left\|\beta(x+n)-\gamma_{2}\right\|^{2}
$$

By Lemma 2.1, it suffices to find an exceptional set with $\left|E_{N, \delta}\right| \leq \frac{\delta}{2}$, such that

$$
\frac{1}{N^{\gamma}} \sum_{n=0}^{N-1} \frac{1}{A_{n}(x)} \lesssim \delta, t, \alpha, \beta 1
$$

for each $x \in[0,1] \backslash E_{N, \delta}$.

We first analyze the case when $\alpha / \beta$ is Diophantine. We distinguish two subcases. First, let us analyze the case $\alpha+t \beta \neq 0$. In this case,

$\left\|\alpha(x+n)-\gamma_{1}+t\left\langle\beta(x+n)-\gamma_{2}\right\rangle\right\|=\left\|(\alpha+t \beta) x+(\alpha+t \beta) n-\gamma_{1}-t \gamma_{2}-t\left[\beta(x+n)-\gamma_{2}\right]+m t\right\|$, where $m=-1$ if $\left\{\beta(x+n)-\gamma_{2}\right\}>1 / 2$ and $m=0$ otherwise. Note that the set of points

$$
S:=
$$

$\left\{(\alpha+t \beta) n-\gamma_{1}-t \gamma_{2}-t\left[\beta(x+n)-\gamma_{2}\right]+m t: x \in[0,1], 0 \leq n \leq N-1, m \in\{0,-1\}\right\}$ has $O_{\beta}(N)$ elements. (21) implies the existence of $E_{N, \delta}$ with $\left|E_{N, \delta}\right|<\delta / 2$ and

$$
\frac{1}{N} \sum_{y \in S} \frac{1}{\|(\alpha+t \beta) x+y\|} \lesssim_{\delta, \alpha, \beta} \log N
$$

for each $x \in[0,1] \backslash E_{N, \delta}$. Thus, (23) follows, even with $N \log N$ instead of $N^{\gamma}$ in the denominator.

Let us now analyze the subcase $\alpha+t \beta=0$. Now,

$$
\left\|\alpha(x+n)-\gamma_{1}+t\left\langle\beta(x+n)-\gamma_{2}\right\rangle\right\|=\left\|-\gamma_{1}-t \gamma_{2}+m t+\frac{\alpha}{\beta}\left[\beta(x+n)-\gamma_{2}\right]\right\|,
$$

where $m$ is as before. Let $\xi$ be either $\gamma_{1}+t \gamma_{2}$ or $\gamma_{1}+t \gamma_{2}+t$. Let

$$
M:=(N \beta)^{\frac{(\gamma-1)(\gamma+\epsilon)}{\gamma+\epsilon-1}} D_{\epsilon}^{\frac{1}{\gamma+\epsilon-1}} .
$$

From Lemma 4.2 we have that for each $x \in[0,1)$

$$
\frac{1}{N^{\gamma}} \sum_{\substack{n=0 \\\left\|\frac{\alpha}{\beta}\left[\beta(x+n)-\gamma_{2}\right]-\xi\right\| \geq \frac{1}{M}}}^{N-1} \frac{1}{\left\|\frac{\alpha}{\beta}\left[\beta(x+n)-\gamma_{2}\right]-\xi\right\|} \lesssim \beta \frac{1}{N^{\gamma}} \sum_{\substack{n=0 \\\left\|\frac{\alpha}{\beta} n-\xi\right\| \geq \frac{1}{M}}}^{N \beta} \frac{1}{\left\|\frac{\alpha}{\beta} n-\xi\right\|} \lesssim 1 .
$$


Let $S(\xi)$ be the set of those $0 \leq n \leq N-1$ such that $\left\|\frac{\alpha}{\beta} n-\xi\right\| \leq \frac{1}{M}$. It was proved in Lemma 4.2 that $S(\xi)$ has at most $N\left(\frac{1}{M D_{\epsilon}}\right)^{\frac{1}{\gamma+\epsilon}}$ points. This is $O_{\alpha, \beta}\left(N^{1 / 10}\right)$, since $\frac{\epsilon}{\gamma+\epsilon-1} \leq \frac{1}{10}$. Define

$$
E_{N, \delta}:=\left\{x \in[0,1]:\left\|\alpha(x+n)-\gamma_{1}\right\| \lesssim \alpha, \beta \delta N^{-\frac{1}{10}} \text { for some } n \in S(\xi)\right\},
$$

and note that $\left|E_{N, \delta}\right| \leq \frac{\delta}{2}$. For $n \in S(\xi)$ we will use the alternative estimate

$$
A_{n}(x) \geq\left\|\alpha(x+n)-\gamma_{1}\right\|^{2} .
$$

Thus, if $x \notin E_{N, \delta}$, then $A_{n}(x) \gtrsim \delta^{2} N^{-\frac{1}{5}}$, and thus

$$
\frac{1}{N^{\gamma}} \sum_{\substack{n=0 \\ n \in S(\xi)}}^{N-1} \frac{1}{A_{n}(x)} \lesssim_{\alpha, \beta} \frac{1}{N^{\gamma}} \delta^{-2} N^{\frac{1}{5}} N^{\frac{1}{10}} \lesssim_{\delta, \alpha, \beta} 1 .
$$

The result now follows from (24) and (25).

Finally, assume that $\alpha / \beta$ is Liouville. Then the result follows right away from (22) and the fact that $A_{n}(x) \geq\left\|\alpha(x+n)-\gamma_{1}\right\|^{2}$.

We now begin the final part of the argument of part (a) of Theorem 1.4. Let now $\gamma$ satisfy (19) and (20) if $\alpha / \beta$ is Diophantine, and let $\gamma=2$ if $\alpha / \beta$ is Liouville.

Let $S \subset[0,1)$ be a set that satisfies (5)-(7), where now $C=0$ (thus minimal decay is assumed). Note that since $f$ is no longer assumed to be continuous, all we can guarantee about $S$ is that it has positive measure. This however is enough to guarantee -via a classical result- that $1_{S} * 1_{-S}(0)>0$. Since $1_{S} * 1_{-S}$ is continuous, there must exist an interval $I$ centered at the origin and $\delta>0$, such that $1_{S} * 1_{-S}(p)>\delta$ on $I$. This implies that for each $p \in-I$,

$$
|\{x \in S: x+p \in S\}|>\delta .
$$

It is automatic (by pigeonholing as before) that there exists a sequence of integers $N_{k} \rightarrow \infty$ and $D=D(\alpha, \beta, s) \in(0, \infty)$ such that

(i) $\left\{P_{k}:=\frac{N_{k}}{\beta}\right\}<|I| / 2$,

(ii) $N_{k}^{\gamma}\left\|N_{k} \frac{\alpha}{\beta}\right\| \leq D$

Let $E_{\left[P_{k}\right], \delta}$ be the exceptional set guaranteed by Lemma 4.3. This set will depend on $k$, but this dependence will not be relevant. Then the set $S_{k}:=\left\{x \in S: x-\left\{P_{k}\right\} \in\right.$ $S\} \cap\left([0,1] \backslash E_{\left[P_{k}\right], \delta}\right)$ is nonempty for each $k \geq 1$. Pick some $x_{k} \in S_{k}$, and finish the argument exactly like in the proof of Theorem 1.3, by working on the orbits of $x_{k}$ and $x_{k}-\left\{P_{k}\right\}$. In particular, note that by the argument in Proposition 2.4,

$$
\left|\prod_{n=0}^{\left[P_{k}\right]-1} P\left(x_{k}-P_{k}+n\right)\right| \lesssim\left|\prod_{n=0}^{\left[P_{k}\right]-1} P\left(x_{k}+n\right)\right|,
$$

with an implicit constant depending only on the fundamental parameters.

We caution that some implicit constants will now also depend on $\gamma, \epsilon$ and $\delta$. This is tolerable, since these only depend on the fundamental parameters.

Part (b) of Theorem 1.4 is much simpler. Indeed, if say, $\alpha$ is rational, then

$$
\left\|\alpha(x+n)-\gamma_{1}\right\| \gtrsim_{\delta, \alpha} 1
$$


for each $n \in \mathbb{N}$ and each $x$ outside some $E_{\delta}$ with measure $\leq \delta$. Lemma 2.1 implies that $|p(\alpha(x+n), \beta(x+n))| \gtrsim_{\delta, \alpha} 1$ for each $x \notin E_{\delta}$. In particular, the estimate in Lemma 2.3

$$
\frac{1}{N} \sum_{n=0}^{N-1} \frac{1}{|P(x+n)|} \lesssim \delta, C_{0}, C_{1}, C_{2}, \alpha, \beta 1
$$

holds trivially (this time with $\gamma=1$ ). The rest of the argument is the same as in the proof of Theorem 1.3. One would have to apply the inequality above for $N=N_{k}$ satisfying

$$
\sup _{k} N_{k}\left\|N_{k} \frac{\alpha}{\beta}\right\| \lesssim 1
$$

\section{Proof of Theorem 1.5}

The proof of part (a) of Theorem 1.5 follows the same general pattern as the proof of Theorem 1.4, but it is significantly simpler. We briefly sketch the details for part (a). As before, given $s>0$, there exists a sequence $N_{k} \rightarrow \infty$ of positive integers satisfying

(i) $\left\{P_{k}:=\frac{N_{k}}{\beta}\right\}<s$,

(ii) $\sup _{k} N_{k} \log N_{k}\left\|N_{k} \frac{\alpha}{\beta}\right\| \lesssim 1$

We have the following analogue of Proposition 2.4.

Proposition 5.1. Let $\left(N_{k}\right)$ be a sequence such that (ii) above holds, and define $P_{k}:=\frac{N_{k}}{\beta}$. Let $P(x)=A+B e(\alpha x), Q(x)=C+D e(\beta x), R(x)=\frac{P(x)}{Q(x)}$. Given $\delta>0$, there exists $E_{k, \delta} \subset[0,1]$ such that $\left|E_{k, \delta}\right| \leq \delta$ such that for each $x, y$ satisfying $x \in[0,1) \backslash E_{k, \delta}$ and $x=y+P_{k}$, we have

$$
\left|\prod_{n=0}^{\left[P_{k}\right]-1} R(y+n)\right| \lesssim A, B, C, D, \alpha, \beta, \delta\left|\prod_{n=0}^{\left[P_{k}\right]-1} R(x+n)\right| .
$$

Proof. Lemma 2.3 implies that there exists an exceptional set $E_{k, \delta} \subset[0,1]$ such that

$$
\frac{1}{N_{k}} \sum_{n=0}^{N_{k}} \frac{1}{|P(x+n)|}+\frac{1}{N_{k}} \sum_{n=0}^{N_{k}} \frac{1}{|Q(x+n)|} \lesssim_{\delta, \alpha, \beta, A, B, C, D} \log N_{k},
$$

for each $x \in[0,1] \backslash E_{k, \delta}$. If $x=y+P_{k}$, we have

Thus, for each $n \in \mathbb{N}$

$$
\begin{aligned}
& |e(\alpha x)-e(\alpha y)|=\left|e\left(P_{k} \alpha\right)-1\right| \lesssim \frac{1}{P_{k} \log P_{k}} \\
& |e(\beta x)-e(\beta y)|=\left|e\left(P_{k} \beta\right)-1\right| \lesssim \frac{1}{P_{k} \log P_{k}} .
\end{aligned}
$$

$$
\begin{aligned}
& |P(y+n)| \leq|P(x+n)|+O\left(\frac{1}{P_{k} \log P_{k}}\right), \\
& |Q(y+n)| \geq|Q(x+n)|-O\left(\frac{1}{P_{k} \log P_{k}}\right) .
\end{aligned}
$$

Use the fact $a+b \leq a e^{\frac{b}{a}}$ for each $a, b>0$ to get

$$
|R(y+n)| \leq|R(x+n)| e^{\frac{1}{P_{k} \log P_{k}|P(x+n)|}+\frac{1}{P_{k} \log P_{k}|Q(x+n)|}} .
$$


The result now follows from (26).

The rest of the argument for part (a) is like in the proof of Theorem 1.4.

The almost periodicity argument is ineffective for part (b) of Theorem 1.5, because of (32). Our argument combines instead the conjugates trick from the proof Theorem 1.3 for $(2,2)$ configurations, with a periodicity argument. Assume there is a measurable function $f: \mathbb{R} \rightarrow \mathbb{C}$, some $d \in(0, \infty)$ and some $S \subset[0,1]$ with positive measure such that

$$
\begin{gathered}
d^{-1}<|f(x)|<d \text { for each } x \in S, \\
\lim _{\substack{|n| \rightarrow \infty \\
n \in \mathbb{Z}}} f(x+n)=0, \\
f(x+1)(A+B e(\alpha x))=f(x)(C+D e(\beta x)),
\end{gathered}
$$

for a.e. $x$, for some fixed $A, B, C, D \in \mathbb{C}, \alpha, \beta \in \mathbb{R}$, none of them zero. Let as before

$$
P(x)=A+B e(\alpha x), \quad Q(x)=C+D e(\beta x) .
$$

Assume also that $\beta=\frac{p}{r}$ is rational. We can trivially assume $A$ is real and that $S+\mathbb{Z}$ contains no zeros of $P$ and $Q$.

Let $B=r e(\theta)$, with $r>0$. By invoking Birkhoff's pointwise ergodic theorem for the function $1_{S}$, there exists $x \in S$ and some $n^{\prime} \in \mathbb{N}$ large enough such that $z:=\left\{-x-\frac{2 \theta}{\alpha}+n^{\prime} \alpha^{-1}\right\} \in S$ and such that $m:=\left[-x-\frac{2 \theta}{\alpha}+n^{\prime} \alpha^{-1}\right]>0$. Let $y:=-x-\frac{2 \theta}{\alpha}+n^{\prime} \alpha^{-1}$. We point out that $x, z, m$ are fixed for the rest of the argument, and thus they can be thought of as a fundamental parameters. The point of this selection is that for each $n \in \mathbb{Z}, A+B e(\alpha y-n \alpha)$ and $A+B e(\alpha x+n \alpha)$ are complex conjugates and thus, for each $N$

$$
\prod_{n=-N+m}^{m}|P(z+n)|=\prod_{n=0}^{N}|P(x+n)| .
$$

It follows that

$$
\prod_{-N+m}^{n=-1}|P(z+n)| \gtrsim_{m, A, B} \prod_{n=0}^{N}|P(x+n)|
$$

Define $T(x)=|Q(x) Q(x+1) \ldots Q(x+r-1)|$. We distinguish two cases.

If $|T(x)| \geq|T(z)|$, then since $Q$ is $r$ - periodic and $\inf _{k \in \mathbb{Z}}|Q(z+k)|>0$, we have

$$
\prod_{n=-N+m}^{-1}|Q(z+n)| \lesssim_{m, x, z, C, D} \prod_{n=0}^{N}|Q(x+n)| .
$$

Using the fact that for each $N>m$

$$
\begin{gathered}
|f(x+N+1)|=|f(x)| \frac{\prod_{n=0}^{N}|Q(x+n)|}{\prod_{n=0}^{N}|P(x+n)|} \\
|f(z-N+m)|=|f(z)| \frac{\prod_{-N+m}^{n=-1}|P(z+n)|}{\prod_{-N+m}^{n=-1}|Q(z+n)|},
\end{gathered}
$$

it follows that (27)-(30) can not hold simultaneously (just let $N \rightarrow \infty$ ).

If $|T(x)| \leq|T(z)|$, then since $Q$ is $r$ - periodic and $\inf _{k \in \mathbb{Z}}|Q(z+k)|>0$, we have 


$$
\prod_{n=-N+m}^{-1}|Q(x+n)| \lesssim_{m, C, D, x, z} \prod_{n=0}^{N}|Q(z+n)| .
$$

Note also that as before,

$$
\prod_{-N+m}^{n=-1}|P(x+n)| \gtrsim_{m, A, B, x, z} \prod_{n=0}^{N}|P(z+n)| .
$$

Using the fact that for each $N \geq m$

$$
\begin{gathered}
|f(z+N+1)|=|f(z)| \frac{\prod_{n=0}^{N}|Q(z+n)|}{\prod_{n=0}^{N}|P(z+n)|} \\
|f(x-N+m)|=|f(x)| \frac{\prod_{-N+m}^{n=-1}|P(x+n)|}{\prod_{-N+m}^{n=-1}|Q(x+n)|},
\end{gathered}
$$

the contradiction is forced like in the previous case.

\section{Open questions}

The argument from Theorem 1.3 seems to be too weak to tackle $(1,4)$ configurations like $(0,0),(1,0),(1, \alpha),(1, \beta),(1, \gamma)$. This is because the best one can guarantee in general is the existence of arbitrarily large $P$ such that $\max \{\|P \alpha\|,\|P \beta\|,\|P \gamma\|\} \lesssim$ $\frac{1}{\sqrt{P}}$. It is not clear whether working with 3 or more orbits would have more to say about this case.

One can wonder if continuity can be removed from the proof of Theorem 1.3 for special $(2,2)$ configurations. If yes, Conjecture 1.1 would follow right away for arbitrary $(2,2)$ configurations. If no continuity is assumed, then the following is a typical worst case scenario

$$
f(x+1)(1+e(\alpha x))=f(x)(1+e(\beta x)),
$$

with $\alpha, \beta$ distinct irrationals. The almost periodicity argument is ineffective in this case. Indeed, it is easy to see that

$$
\lim _{N \rightarrow \infty} \inf _{x \in[0,1]} \frac{1}{N} \sum_{n=1}^{N} \frac{1}{|1-e(x+\alpha n)|}=\infty .
$$

To see this, recall that by Dirichlet's theorem, for each $N$ there exists $p_{N} \in \mathbb{N}$ and $1 \leq m_{N} \leq N$ relatively prime, such that

$$
\left|\alpha-\frac{p_{N}}{m_{N}}\right| \leq \frac{1}{m_{N} N}
$$

For each $i \in\left\{0,1, \ldots, m_{N}-1\right\}$, roughly $N / m_{N}$ of the points $1 \leq n \leq N$ satisfy

$$
\left\|\alpha n-\frac{i}{m_{N}}\right\| \leq \frac{1}{m_{N}} \text {. }
$$

Thus, for each $x \in[0,1]$

$$
\frac{1}{N} \sum_{n=1}^{N} \frac{1}{|1-e(x+\alpha n)|} \gtrsim \sum_{i=1}^{m_{N}} \frac{1}{i} \sim \log m_{N} .
$$


Finally, note that the irrationality of $\alpha$ forces $m_{N} \rightarrow \infty$.

It would be very interesting to know if given $\alpha \neq \beta$ the recurrence (31) can even hold along the orbit of a single point $x_{0} \in[0,1]$, in such a way that $\lim _{|N| \rightarrow \infty} f\left(x_{0}+N\right)=0$.

\section{Acknowledgements}

We would like to thank Christoph Thiele for stimulating our initial interest on the HRT conjecture. Special thanks to Zubin Gautam, Nets Katz, Joseph Rosenblatt and Alexandru Zaharescu for helpful discussions on the subject.

\section{References}

[1] M. Bownik and D. Speegle, Linear independence of Parseval wavelets (2009). Preprint.

[2] C. Demeter and Z. Gautam, On the finite linear independence of lattice Gabor systems (2010). Preprint.

[3] C. Heil, Linear independence of finite Gabor systems, in Harmonic analysis and applications, Appl. Numer. Harmon. Anal., 171-206, Birkhäuser Boston, Boston, MA (2006).

[4] C. Heil, J. Ramanathan, and P. Topiwala, Linear independence of time-frequency translates, Proc. Amer. Math. Soc. 124 (1996), no. 9, 2787-2795.

[5] P. A. Linnell, von Neumann algebras and linear independence of translates, Proc. Amer. Math. Soc. 127 (1999), no. 11, 3269-3277.

[6] I. Niven and H. S. Zuckerman, An introduction to the theory of numbers, Second edition, John Wiley \& Sons Inc., New York (1966).

Department of Mathematics, Indiana University, 831 East 3rd St., Bloomington in 47405

E-mail address: demeterc@indiana.edu 